\title{
Aplicação do refinamento das regras de ligação da CIF à Escala Visual Analógica e aos questionários Roland Morris e SF-36
}

Rafaella Stradiotto Bernardelli (http://orcid.org/0000-0002-4613-0834) ${ }^{1}$

Bárbara Cordeiro Santos (http://orcid.org/0000-0003-1222-7977) ${ }^{1}$

Karoleen Oswald Scharan (http://orcid.org/0000-0002-4420-3171) ${ }^{1}$

Katren Pedroso Corrêa (http://orcid.org/0000-0002-0175-2728) ${ }^{1}$

Maria Isabel Barboza Silveira (http://orcid.org/0000-0001-7952-8219) ${ }^{1}$

Auristela Duarte de Lima Moser (http://orcid.org/0000-0001-5086-0701) ${ }^{1}$
${ }^{1}$ Programa de Pós-

Graduação de Tecnologia

em Saúde, Pontifícia

Universidade Católica

do Paraná. R. Imaculada

Conceição 1155, Prado

Velho. 80215-901

Curitiba PR Brasil.

rafaellasb@yahoo.com.br
Resumo A Escala Visual Analógica (EVA), o Questionário de Incapacidade de Roland Morris (RMDQ) e Questionário de Qualidade de Vida SF-36, amplamente utilizados, tiveram seu conteúdo conectado à CIF por regras propostas em 2002 e 2005. Em 2016 foram refinadas e ainda não foram aplicadas. Aplicar as regras de conexão de conteúdo refinadas para os instrumentos EVA, RMDQ e SF-36. Dois profissionais de saúde identificaram os conceitos significativos e vincularam às categorias mais específicas da CIF, um terceiro arbitrou divergências. O grau de concordância foi dado pelo coeficiente kappa. Houve alto grau de concordância (Kappa=0,93 p<0,001). O conceito principal da EVA foi conectado à categoria b280, os 24 conceitos principais do RMDQ, à categoria b28013 e os 27 adicionais a outras categorias. $O$ SF-36 teve 36 conceitos principais e 30 adicionais identificados, do total, 17 não foram definíveis pela CIF. Dos conceitos conectados dos 3 instrumentos 39 referem-se à Funções do Corpo, 57 à Atividades e Participação e 4 à Fatores Ambientais. O refinamento das regras propiciou mais clareza no processo de identificar, relacionar o conteúdo dos instrumentos à CIF e expor os resultado e aumentou o número de conceitos identificados e categorias contempladas pelos instrumentos.

Palavras-chave CIF, Regras de ligação, Inquéritos e Questionários 


\section{Introdução}

A dor crônica tem sido reportada com alta prevalência e decorrente de múltiplos fatores ${ }^{1-3}$. Portanto, é necessário considerar os fatores físicos, psicológicos e ambientais envolvidos nessa condição de saúde $e^{4}$.

Uma estrutura universalmente aceita que contempla um modelo biopsicossocial é a Classificação Internacional de Funcionalidade, Incapacidade e Saúde (CIF), que foi criada pela Organização Mundial da Saúde (OMS) e padroniza a linguagem dos estados de saúde dos indivíduos referentes a categorias relacionadas às Funções do Corpo, Estruturas do Corpo, Atividades e Participação e Fatores Ambientais ${ }^{5}$.

$\mathrm{O}$ uso da classificação é recomendado em conjunto com ferramentas da prática clínica para valorizar as informações já coletadas e complementá-las, encorajando os profissionais a selecionar os instrumentos mais apropriados para as suas propostas clínicas ${ }^{6-8}$. Essas ferramentas, entretanto, têm sido utilizadas em uma perspectiva fortemente ancorada em um modelo biomédico de saúde, mesmo quando coletam-se dados referentes a participação social e condições ambientais ${ }^{9}$. Essa abordagem resulta em práticas descontextualizadas, focadas em intervenções e monitoramento de sinais e sintomas.

O conteúdo da CIF, quando conectado a esses instrumentos, irá contribuir para a operacionalização de um conceito de saúde expandido, permitindo a interpretação dos dados no sentido de atribuir o mesmo status aos componentes psicológicos e fatores contextuais, considerando privilegiar a interação e interdependência deles, como produto e produtor de saúde e seus estados relacionados.

Para melhor acurácia nesse processo é recomendado ligar o conteúdo dos instrumentos à CIF, fazendo com que a CIF seja a ferramenta capaz de traduzir a informação contida nos instrumentos ${ }^{10}$. Em vista disso, em 2002 foram propostas as primeiras regras de ligação ${ }^{11}$, em 2005 foram atualizadas ${ }^{6}$ e, em 2016 refinadas $^{10}$.

O refinamento das regras de ligação objetivou aumentar a transparência e a confiabilidade do processo de ligação do conteúdo do instrumento, sendo propostas cinco melhorias. A primeira refere-se à preparação da informação a ser ligada. A regras de ligação de 2002 e 2005 salientavam que o conceito principal a ser escolhido deveria ser identificado antes de começar o processo de ligação. Desse modo, o processo apresentava o risco de aplicação mecânica das regras sem considerar o contexto ou a proposta na qual a informação é coletada.

O refinamento propõe identificar a "proposta da informação" a ser ligada a uma categoria antes de identificar o conceito significativo, para isso deve-se responder às seguintes questões: "essa é a peça-chave da informação? Sobre o que é esse item?". Portanto, as regras propõem não apenas a identificação de um conceito significativo, mas também separar em conceito principal e conceitos adicionais de acordo com a proposta e a perspectiva da informação a ser ligada com a $\mathrm{CIF}^{10}$.

Outro refinamento leva em conta a perspectiva da informação coletada. Propõe a documentação da perspectiva em que a informação foi coletada, pelo instrumento de coleta dos dados ou pela forma de coleta. As perspectivas mais proeminentes são: descritiva, envolvendo a capacidade e o desempenho em realizar alguma tarefa ou atividade; avaliação da satisfação individual em relação a uma determinada situação, perguntando em que medida as expectativas e esperanças pessoais foram alcançadas; e, a perspectiva de necessidade ou dependência, que se refere como vários dispositivos de assistência são necessários para realizar certas tarefas ou atividades.

Nas regras de 2002 e 2005 não havia informação sobre a categorização das opções de resposta capturadas durante o processo de ligação, além dos conceitos significativos ${ }^{6,11}$. O refinamento propõe identificar e documentar a categorização das opções de resposta como: intensidade, frequência, duração, confirmação ou concordância, e atributos qualitativos. Essa informação é relevante apenas a questionários, avaliações ou testes que contemplem opções de respostas. É válido mencionar que existem opções de respostas em que não é possível estabelecer a ligação ou que a própria resposta contenha conceitos significativos $^{10}$.

As regras propostas em 2002 e atualizadas em 2005 recomendam que os qualificadores 8 (não especificado) e 9 (não aplicável) não fossem uti$\operatorname{lizados}^{6,11}$. O uso desses qualificadores são recomendados no refinamento, uma vez que a experiência mostrou que não utilizar essa categorização implica em perda de informação. Se um conceito é ligado ao qualificador 8 ou 9, informações adicionais não especificadas ou não aplicáveis pela CIF devem ser documentadas juntamente com as categorias da $\mathrm{CIF}^{10}$.

A última proposta orienta que à informação não contida na CIF deve ser atribuída a abreviação nc (não coberto), embora o processo de ligação possa ser aplicado a qualquer tipo de in- 
formação em saúde, nem sempre é possível ligar uma informação a uma categoria da CIF. Esse deve ser o caso da informação a ser ligada estar além do escopo da CIF ou talvez ser muito específica para ser ligada à classificação ${ }^{10}$.

Muitos instrumentos são usados em pesquisa e na prática clínica para avaliar dor, funcionalidade e qualidade de vida. Aqueles que mais aparecem na literatura são a Escala Visual Análoga (EVA $)^{12}$, o Questionário de Incapacidade de Roland Morris, Roland Morris Disability Questionnaire (RMDQ $)^{13}$ e o questionário de qualidade de vida, Short Form Health Survey (SF-36) ${ }^{14}$, usados para acessar a dor do paciente, sua capacidade funcional e a qualidade de vida, respectivamente.

Esses instrumentos já tiveram seu conteúdo ligado à CIF por estudos que aplicaram as regras de 2002 e 2005, como Prodinger et al. ${ }^{15}$, Sigl et al. ${ }^{16}$, Fréz et al. ${ }^{17}$, Schepers et al. ${ }^{18}$, Geyh et al. ${ }^{19}$ e Cieza e Stucki ${ }^{7}$.

Cieza et al. ${ }^{10}$ sugerem que pesquisadores na área apliquem a versão atualizada das regras e comentem sobre as experiências durante o processo de utilização delas. É nesse sentido que o presente estudo almeja contribuir. Até o momento, nenhum estudo foi identificado na literatura que tenha sido conduzido com a aplicação dos refinamentos.

Considerando a magnitude das mudanças qualitativas trazidas pelas novas regras, e o grau de relevância dos instrumentos adotados neste estudo na prática clínica dos profissionais de saúde, os autores consideram importante aplicar as novas regras a eles.

Assim, o objetivo do presente estudo foi aplicar os refinamentos das regras de ligação à EVA, RMDQ e SF-36 propostos na literatura.

\section{Método}

O processo de ligação de conteúdo dos instrumentos ocorreu por meio da aplicação do refinamento das regras de ligação proposto por Cieza et al. ${ }^{10}$. Participaram deste processo dois profissionais de saúde brasileiros com curso de CIF certificado por centro colaborador da OMS e que já tinham experiência com processo de ligação com as regras anteriores. Eles também incluíram em um grupo de estudo em CIF a ferramenta e-learning tool, disponibilizada e recomendada pela OMS. Um terceiro pesquisador fisioterapeuta com experiência semelhante foi consultado em caso de divergências.
De acordo com o guia metodológico ${ }^{10}$, a proposta da informação a ser ligada foi identificada antes da identificação do conceito significativo. Então, os dois profissionais de saúde identificaram os conceitos significativos contidos nos instrumentos considerando o contexto, afirmações e as opções de resposta para então selecionar qualquer outro conceito adicional. Em seguida os conceitos significativos foram comparados para se obter consenso.

Outro refinamento utilizado no presente estudo foi identificar e descrever as perspectivas adotadas nos instrumentos. As questões foram identificadas e descritas como descritivas, de avaliação e necessidade ou dependência. A categorização das opções de resposta ocorreu pela identificação e documentação como intensidade, frequência, duração, confirmação ou concordância e atributos qualitativos.

Na sequência, cada conceito significativo foi ligado à categoria mais precisa da CIF. No caso de relação de conceitos por conjunção, eles também foram registrados. Os conceitos significativos relacionados a saúde física, saúde mental, saúde em geral, incapacidade em geral, funcionalidade e desenvolvimento infantil e que não provessem informação suficiente para selecionar uma categoria da CIF foram classificados como não definíveis e denominados como as nd-sf, nd-sm, nd-sg, nd-inc, nd-func, e nd-des, respectivamente. Quando um conceito significativo foi identificado como um fator pessoal foi denominado como fp e, quando não foi coberto pela CIF foi denominado como nc, como diagnóstico ou condição de saúde (nc-cs) e qualidade de vida em geral (nc-qv) ${ }^{10}$. Essas situações são chamadas de casos especiais.

O processo de ligação foi organizado em planilha do Microsoft Excel 2007. O grau de concordância entre os dois profissionais de saúde em relação a identificação e ligação dos conceitos foram calculados pelo Kappa statistic ${ }^{20}$. Todos os critérios pré-determinados para verificar concordância interexaminador pelo coeficiente Cohen's Kappa foram seguidos neste estudo. Por exemplo, a variável estava em sua natureza nominal (categorias da CIF), a comparação foi realizada apenas entre dois pesquisadores e não houve dados omissos ${ }^{21-26}$. Ademais, esse é o coeficiente recomendado pelas regras de ligação e tem sido frequentemente reportado na literatura como o escolhido para avaliar o grau de concordância entre profissionais na identificação de conceitos significativos e na ligação desses conceitos com as 
categorias da $\mathrm{CIF}^{6,10,16,27-29}$. O coeficiente de correlação Kappa varia entre 0 e 1, em que 0 indica nenhuma concordância além da esperada ao acaso e 1 indica concordância perfeita. Coeficientes Kappa acima de 0,61 são considerados como boa concordância ${ }^{18}$. A análise estatística foi realizada no software SPSS 22.0.

\section{Resultados}

Para a apresentação dos resultados foi usado o modelo proposto pelo guia metodológico. Um quadro foi gerado contendo as informações extraídas dos instrumentos conjuntamente com os resultados do processo de ligação, a perspectiva adotada na informação, a classificação das opções de resposta e as categorias dos conceitos principais e conceitos adicionais. Os resultados do conteúdo ligado aos instrumentos estão descritos nos Quadros 1 e 2.

Houve quase concordância total entre os pesquisadores na ligação dos itens do RMQD e SF36 e as categorias da CIF (Índice de Kappa=0,93 $\mathrm{p}<0,001)$.

A Tabela 1 mostra os 10 conceitos significativos do SF-36 e 1 do RMDQ que foram ligados aos casos especiais.

A Tabela 2 mostra o número total de conceitos identificados nos instrumentos e as categorias da CIF ligadas a esses conceitos. Em relação ao RMDQ, foi considerado que todos os itens eram embasados no conceito dor nas costas, e que foi considerado o conceito principal de todos os itens e ligado à uma categoria (b28013). Nos 23 itens dos 24 no RMDQ, foi possível identificar 27 conceitos adicionais, a maior parte deles ligados a categorias do componente de Atividades e Participação, seguido de Funções do Corpo e Fatores ambientais (Quadro 1 e Tabela 2).

Em relação ao SF-36, foi observado que dos 36 conceitos principais, 13 não foram definidos pela CIF, e, dos 30 conceitos adicionais, 4 não foram definíveis (Tabela 1). Isso mostra que a CIF não contempla conceitos abrangentes relacionados a saúde geral, saúde física, saúde mental e condições de saúde.

As questões 4 e 5 do SF-36 trouxeram informações relevantes já no cabeçalho e, dessa informação, para cada questão um conceito principal e conceitos adicionais foram identificados. Cada item dessas questões permitiu identificar informações adicionais.

Esses resultados permitem a comparação entre as aplicações das regras de 2002, 2005 e 2016 quando aplicadas aos mesmos instrumentos permitindo discussões. A Tabela 3 apresenta os resultados numéricos da ligação dos três instrumentos da CIF no presente estudo e de estudos anteriores. A aplicação do refinamento das regras de ligação proporcionou um aumento nos conceitos identificados e ligados do SF-36 à CIF. Para ambos, SF36 e RMDQ houve um aumento no número de categorias. Para a EVA os resultados foram os mesmos no presente estudo e para Scheuringer et al. ${ }^{30}$.

Para aumentar a transparência e a confiabilidade durante o processo de ligação, seguimos a sugestão de colocar os resultados em uma tabela contendo as informações extraídas dos instrumentos, juntamente com os resultados do processo de ligação, valorizando a perspectiva adotada nas informações, a classificação das opções de resposta e as categorias dos conceitos principais e adicionais.

\section{Discussão}

O estudo objetivou reportar a experiência de aplicar o refinamento das regras de ligação proposto na literatura. Essa proposta ${ }^{10}$, que sugere uma reflexão antes da identificação de conceitos nos guiou à identificação de um conceito principal relacionado a cada item dos instrumentos e à identificação de conceitos adicionais e informações adicionais relacionadas aos itens.

Os itens "a" e " $\mathrm{e}$ " das regras prévias ${ }^{6}$ enfatizavam que a informação deveria ser identificada antes de começar o processo de ligação. Porém, havia um risco de da informação ser extraída mecanicamente sem considerar seu contexto ou a proposta da informação coletada ${ }^{10}$. Nesse caso, a informação poderia ser mascarada ao se identificar categorias sem definir os itens mais representativos do instrumento. As regras recentes propõem a identificação da informação a ser ligada antes da identificação dos conceitos principais e conceitos adicionais ${ }^{10}$.

Depois da aplicação da regra dois $^{10}$, para identificar o conceito principal, a regra três foi aplicada para identificar qualquer conceito adicional à informação principal. Esses conceitos também poderiam ser identificados a partir das opções de resposta. A organização dos conceitos principais e adicionais irá permitir uma comparação de informação em saúde de uma forma mais específica. Um exemplo para ilustrar isso ocorreu no item 3 do SF-36 para o estudo de Cieza et al. ${ }^{11}$ e o presente estudo. Embora o mes- 
Quadro 1. Extração dos conteúdos da EVA e do RMDQ.

\begin{tabular}{|c|c|c|c|c|c|c|c|c|c|}
\hline Cabeçalho & Item & $\begin{array}{l}\text { Perspectiva } \\
\text { adotada na } \\
\text { informação }\end{array}$ & $\begin{array}{l}\text { Opções } \\
\text { de res- } \\
\text { posta }\end{array}$ & $\begin{array}{c}\text { Classifi- } \\
\text { cação de } \\
\text { opções de } \\
\text { resposta }\end{array}$ & $\begin{array}{c}\text { Con- } \\
\text { ceito } \\
\text { princi- } \\
\text { pal }\end{array}$ & $\begin{array}{l}\text { Conceito } \\
\text { adicional }\end{array}$ & $\begin{array}{c}\text { Categoria } \\
\text { CIF do } \\
\text { conceito } \\
\text { principal }\end{array}$ & $\begin{array}{c}\text { Categorias CIF } \\
\text { dos demais } \\
\text { conceitos }\end{array}$ & $\begin{array}{c}\text { Informação } \\
\text { Adicional }\end{array}$ \\
\hline \multicolumn{10}{|l|}{ EVA } \\
\hline \begin{tabular}{|l|} 
Quanto de \\
dor nas cos- \\
tas você está \\
sentindo \\
\end{tabular} & 1 & Avaliação & 0 a 10 & Intensidade & Dor & & b280 Dor & & \\
\hline \multicolumn{10}{|l|}{ RMDQ } \\
\hline \multirow[t]{8}{*}{$\begin{array}{l}\text { Dores nas } \\
\text { costas }\end{array}$} & 1 & \multirow[t]{7}{*}{$\begin{array}{l}\text { Descritiva: } \\
\text { Desempe- } \\
\text { nho }\end{array}$} & \multirow[t]{8}{*}{$\begin{array}{l}\text { Sim ou } \\
\text { Não }\end{array}$} & \multirow[t]{8}{*}{ Confirmação } & \begin{tabular}{|l} 
Dores \\
nas \\
costas \\
\end{tabular} & Ficar em casa & \begin{tabular}{|l|} 
b28013 \\
Dor nas \\
costas
\end{tabular} & nc & $\begin{array}{l}\text { Maior parte } \\
\text { do tempo }\end{array}$ \\
\hline & 2 & & & & $\begin{array}{l}\text { Dores } \\
\text { nas } \\
\text { costas } \\
\end{array}$ & $\begin{array}{l}\text { Mudar de } \\
\text { posição }\end{array}$ & $\begin{array}{l}\text { b28013 } \\
\text { Dor nas } \\
\text { costas }\end{array}$ & $\begin{array}{l}\text { d410 Mudar a } \\
\text { posição básica do } \\
\text { corpo }\end{array}$ & $\begin{array}{l}\text { Frequente- } \\
\text { mente }\end{array}$ \\
\hline & 3 & & & & $\begin{array}{l}\text { Dores } \\
\text { nas } \\
\text { costas } \\
\end{array}$ & Andar & $\begin{array}{l}\text { b28013 } \\
\text { Dor nas } \\
\text { costas }\end{array}$ & d450 Andar & Mais devagar \\
\hline & 4 & & & & $\begin{array}{l}\text { Dores } \\
\text { nas } \\
\text { costas } \\
\end{array}$ & $\begin{array}{l}\text { Tarefas de } \\
\text { casa }\end{array}$ & $\begin{array}{l}\text { b28013 } \\
\text { Dor nas } \\
\text { costas } \\
\end{array}$ & $\begin{array}{l}\text { d640 Realizar as } \\
\text { tarefas domésticas }\end{array}$ & \\
\hline & 5 & & & & $\begin{array}{l}\text { Dores } \\
\text { nas } \\
\text { costas }\end{array}$ & $\begin{array}{l}\text { Subir esca- } \\
\text { das / Uso do } \\
\text { corrimão }\end{array}$ & $\begin{array}{l}\text { b28013 } \\
\text { Dor nas } \\
\text { costas }\end{array}$ & $\begin{array}{l}\text { d4551 Subir / } \\
\text { e1201 Produtos } \\
\text { e tecnologias de } \\
\text { assistência desti- } \\
\text { nados a facilitar } \\
\text { a mobilidade e o } \\
\text { transporte pessoal } \\
\text { em ambientes in- } \\
\text { ternos e externos } \\
\end{array}$ & \\
\hline & 6 & & & & $\begin{array}{l}\text { Dores } \\
\text { nas } \\
\text { costas } \\
\end{array}$ & Deitar-se & $\begin{array}{l}\text { b28013 } \\
\text { Dor nas } \\
\text { costas }\end{array}$ & $\begin{array}{l}\text { b28013 Dor nas } \\
\text { costas }\end{array}$ & $\begin{array}{l}\text { Com mais fre- } \\
\text { quência para } \\
\text { descansar }\end{array}$ \\
\hline & 7 & & & & $\begin{array}{l}\text { Dores } \\
\text { nas } \\
\text { costas }\end{array}$ & $\begin{array}{l}\text { Levantar-se } \\
\text { / Apoiar em } \\
\text { alguma coisa }\end{array}$ & $\begin{array}{l}\text { b28013 } \\
\text { Dor nas } \\
\text { costas }\end{array}$ & $\begin{array}{l}\text { d4104 Levantar-se } \\
\text { /e1201 Produtos } \\
\text { e tecnologias de } \\
\text { assistência desti- } \\
\text { nados a facilitar } \\
\text { a mobilidade e o } \\
\text { transporte pessoal } \\
\text { em ambientes in- } \\
\text { ternos e externos }\end{array}$ & Poltrona \\
\hline & 8 & $\begin{array}{l}\text { Dependên- } \\
\text { cia }\end{array}$ & & & $\begin{array}{l}\text { Dores } \\
\text { nas } \\
\text { costas }\end{array}$ & $\begin{array}{l}\text { Conseguir } \\
\text { que outras } \\
\text { pessoas } \\
\text { façam as cois- } \\
\text { as por mim } \\
\end{array}$ & $\begin{array}{l}\text { b28013 } \\
\text { Dor nas } \\
\text { costas }\end{array}$ & $\begin{array}{l}\text { e3 Apoio e Rela- } \\
\text { cionamento }\end{array}$ & \\
\hline
\end{tabular}

mo conceito significativo (atividades rigorosas, correr, levantar objetos pesados e participar em esportes extenuantes) tenha sido identificado em ambos os estudos, no estudo de Cieza et al. ${ }^{11}$, eles ligaram de forma independente todos os concei- tos às categorias da CIF referentes ao componente de Atividades e Participação, com exceção do conceito atividades rigorosas, descrito como não definível. O presente estudo identificou o conceito atividades rigorosas como o conceito principal 
Chart 1. VAS, RMDQ linking extraction.

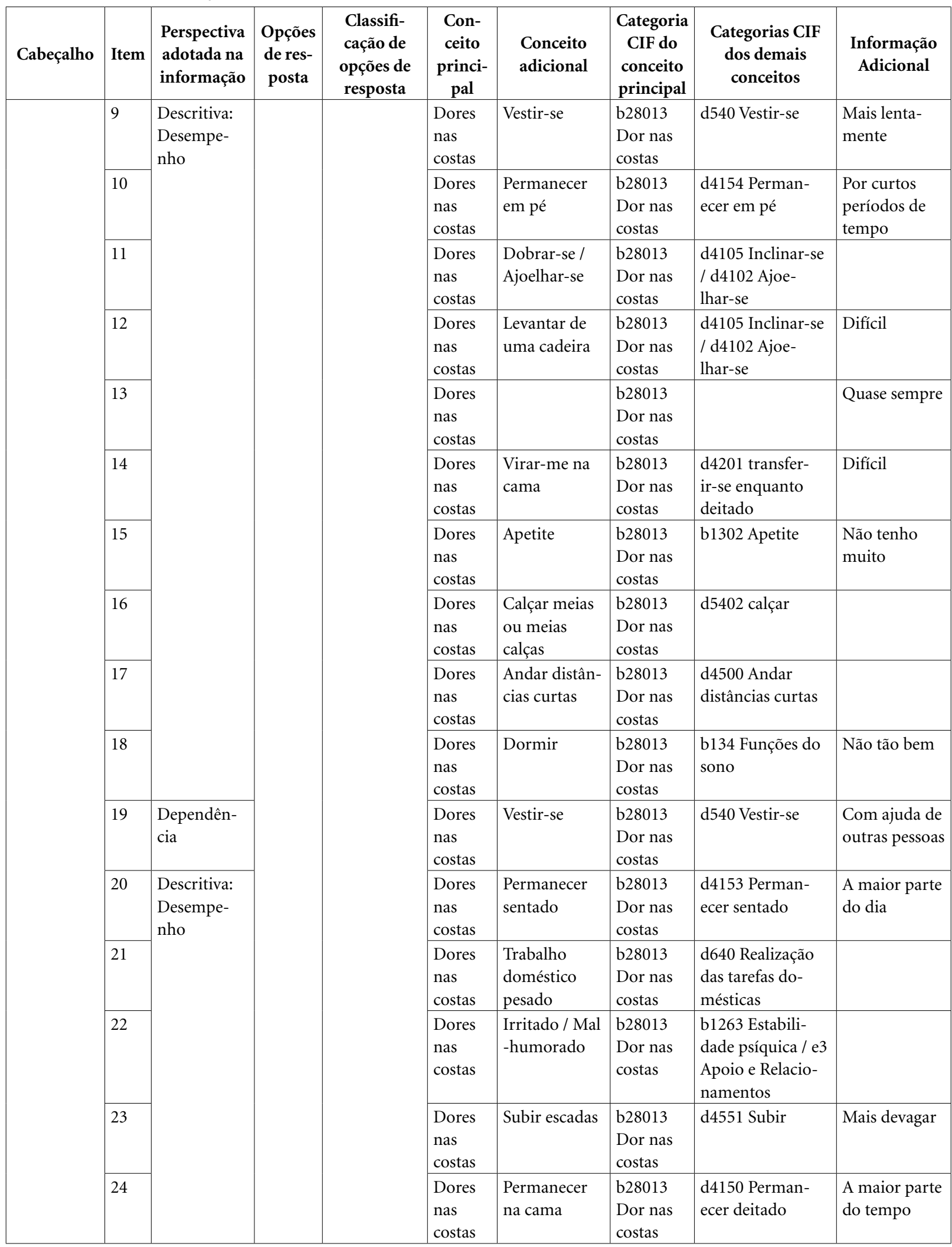

"nc": corresponde a não coberto pela CIF. Fonte: as autoras. 
Quadro 2. Extração dos conteúdos do SF-36.

\begin{tabular}{|c|c|c|c|c|c|c|c|c|c|}
\hline Cabeçalho & Item & $\begin{array}{c}\text { Pers- } \\
\text { pectiva } \\
\text { adotada } \\
\text { na infor- } \\
\text { mação }\end{array}$ & $\begin{array}{l}\text { Opções de } \\
\text { resposta }\end{array}$ & $\begin{array}{l}\text { Classifi- } \\
\text { cação de } \\
\text { opções } \\
\text { de res- } \\
\text { posta }\end{array}$ & $\begin{array}{c}\text { Conceito } \\
\text { princi- } \\
\text { pal }\end{array}$ & $\begin{array}{l}\text { Conceito } \\
\text { adicional }\end{array}$ & $\begin{array}{l}\text { Categoria } \\
\text { CIF do con- } \\
\text { ceito prin- } \\
\text { cipal }\end{array}$ & $\begin{array}{l}\text { Categorias } \\
\text { CIF dos } \\
\text { demais con- } \\
\text { ceitos }\end{array}$ & $\begin{array}{c}\text { Informação } \\
\text { Adicional }\end{array}$ \\
\hline \multicolumn{10}{|l|}{ SF-36 } \\
\hline $\begin{array}{l}\text { Em geral, você } \\
\text { diria que sua } \\
\text { saúde é: }\end{array}$ & 1 & \multirow[t]{2}{*}{ Appraisal } & $\begin{array}{l}\text { Excelente } \\
(1) ; \\
\text { Muito Boa } \\
(2) ; \\
\text { Boa (3); } \\
\text { Ruim (4); } \\
\text { Muito Ruim } \\
\text { (5) }\end{array}$ & $\begin{array}{l}\text { Intensi- } \\
\text { dade }\end{array}$ & $\begin{array}{l}\text { Saúde } \\
\text { Geral }\end{array}$ & & nd-sg & & \\
\hline $\begin{array}{l}\text { Comparada há } \\
\text { um ano atrás, } \\
\text { como você } \\
\text { classificaria sua } \\
\text { saúde em geral } \\
\text { agora. }\end{array}$ & 2 & & $\begin{array}{l}\text { Muito Mel- } \\
\text { hor (1); } \\
\text { Um Pouco } \\
\text { Melhor (2); } \\
\text { Quase a } \\
\text { mesma (3); } \\
\text { Um Pouco } \\
\text { Pior (4); } \\
\text { Muito Pior } \\
\text { (5). }\end{array}$ & $\begin{array}{l}\text { Intensi- } \\
\text { dade }\end{array}$ & Saúde & & nd-sg & & \\
\hline \multirow{2}{*}{$\begin{array}{l}\text { Os seguintes } \\
\text { itens são sobre } \\
\text { atividades que } \\
\text { você poderia } \\
\text { fazer atual- } \\
\text { mente durante } \\
\text { um dia co- } \\
\text { mum. Devido } \\
\text { à sua saúde, } \\
\text { você teria } \\
\text { dificuldade } \\
\text { para fazer estas } \\
\text { atividades? } \\
\text { Neste caso, } \\
\text { quando? }\end{array}$} & $3 a$ & \multirow[t]{2}{*}{$\begin{array}{l}\text { Des- } \\
\text { critiva: } \\
\text { Desem- } \\
\text { penho }\end{array}$} & $\begin{array}{l}\text { Sim, dificulta } \\
\text { muito (1); } \\
\text { Sim, dificulta } \\
\text { um pouco } \\
(2) ; \\
\text { Não, não } \\
\text { dificulta de } \\
\text { modo algum } \\
(3) \text {. }\end{array}$ & \multirow[t]{2}{*}{$\begin{array}{l}\text { Intensi- } \\
\text { dade }\end{array}$} & $\begin{array}{l}\text { Ativi- } \\
\text { dades } \\
\text { vigorosas }\end{array}$ & $\begin{array}{l}\text { Correr/ } \\
\text { Levantar } \\
\text { objetos } \\
\text { pesados } \\
\text { / Partic- } \\
\text { ipar em } \\
\text { esportes } \\
\text { árduos }\end{array}$ & $\begin{array}{l}\text { d Ativi- } \\
\text { dades e } \\
\text { Participação } \\
\text { (atividades } \\
\text { vigorosas) }\end{array}$ & $\begin{array}{l}\text { d4552 Cor- } \\
\text { rer / d4300 } \\
\text { Levantar } \\
\text { objetos / } \\
\text { d9201 Prati- } \\
\text { car esportes }\end{array}$ & $\begin{array}{l}\text { Esportes árdu- } \\
\text { os, vigorosos }\end{array}$ \\
\hline & $3 b$ & & & & \begin{tabular}{|l|} 
Ativi- \\
dades \\
modera- \\
das
\end{tabular} & $\begin{array}{l}\text { moving } \\
\text { a table/ } \\
\text { pushing } \\
\text { a vacuum } \\
\text { cleaner/ } \\
\text { bowling/ } \\
\text { sweeping }\end{array}$ & $\begin{array}{l}\text { d Ativi- } \\
\text { dades e } \\
\text { Participação } \\
\text { (atividades } \\
\text { moderadas) }\end{array}$ & $\begin{array}{l}\text { d430 Levan- } \\
\text { tar e carregar } \\
\text { objetos/ } \\
\text { d6403 } \\
\text { Utilizar } \\
\text { aparelhos } \\
\text { domésticos/ } \\
\text { d9201 Prati- } \\
\text { car esportes/ } \\
\text { d6402 Lim- } \\
\text { par a habi- } \\
\text { tação }\end{array}$ & $\begin{array}{l}\text { Jogar bola, } \\
\text { moderada }\end{array}$ \\
\hline
\end{tabular}

e os demais como conceitos adicionais, considerando o contexto e a perspectiva do item do instrumento como um todo. Isso torna possível ligar esse conceito ao componente de Atividades e Participação, considerando quais conceitos adicionais relacionados ao conceito principal estão ligados às categorias de Atividades e Participação.
Os conceitos principais identificados em cada item dos instrumentos podem variar de acordo com o contexto clínico e do background dos pesquisadores envolvidos. Essa perspectiva está alinhada com o modelo biopsicossocial que considera a relevância das abordagens com base em achados contextualizados. 
Quadro 2. Extração dos conteúdos do SF-36.

\begin{tabular}{|c|c|c|c|c|c|c|c|c|c|}
\hline Cabeçalho & Item & $\begin{array}{c}\text { Pers- } \\
\text { pectiva } \\
\text { adotada } \\
\text { na infor- } \\
\text { mação }\end{array}$ & $\begin{array}{c}\text { Opções de } \\
\text { resposta }\end{array}$ & $\begin{array}{c}\text { Classifi- } \\
\text { cação de } \\
\text { opções } \\
\text { de res- } \\
\text { posta } \\
\end{array}$ & $\begin{array}{c}\text { Conceito } \\
\text { princi- } \\
\text { pal }\end{array}$ & $\begin{array}{r}\text { Conceito } \\
\text { adicional }\end{array}$ & $\begin{array}{l}\text { Categoria } \\
\text { CIF do con- } \\
\text { ceito prin- } \\
\text { cipal }\end{array}$ & $\begin{array}{l}\text { Categorias } \\
\text { CIF dos } \\
\text { demais con- } \\
\text { ceitos }\end{array}$ & $\begin{array}{c}\text { Informação } \\
\text { Adicional }\end{array}$ \\
\hline & $3 c$ & & & & $\begin{array}{l}\text { Levan- } \\
\text { tar e } \\
\text { carregar } \\
\text { manti- } \\
\text { mentos } \\
\end{array}$ & & $\begin{array}{l}\text { d430 Levan- } \\
\text { tar e carregar } \\
\text { objetos }\end{array}$ & & Mantimentos \\
\hline & $3 d$ & & & & Subir & & d4551 Subir & & $\begin{array}{l}\text { Vários lances } \\
\text { de escada }\end{array}$ \\
\hline & $3 e$ & & & & Subir & & d4551 Subir & & $\begin{array}{l}\text { Um lance de } \\
\text { escada }\end{array}$ \\
\hline & $3 \mathrm{f}$ & & & & $\begin{array}{l}\text { Mobili- } \\
\text { dade }\end{array}$ & $\begin{array}{l}\text { Curvar- } \\
\text { se/ Ajo- } \\
\text { elhar-se/ } \\
\text { Dobrar-se }\end{array}$ & $\begin{array}{l}\text { d4 Mobili- } \\
\text { dade }\end{array}$ & \begin{tabular}{|l}
$\mathrm{d} 4101$ \\
Agachar-se/ \\
d4102 Ajoel- \\
har-se/ d4105 \\
Inclinar-se \\
\end{tabular} & \\
\hline & $3 g$ & & & & Andar & & $\begin{array}{l}\text { d4501 Andar } \\
\text { distâncias } \\
\text { longas }\end{array}$ & & $\begin{array}{l}\text { Mais de } 1 \\
\text { quilômetro }\end{array}$ \\
\hline & $3 \mathrm{~h}$ & & & & Andar & & $\begin{array}{l}\text { d4501 Andar } \\
\text { distâncias } \\
\text { longas }\end{array}$ & & $\begin{array}{l}\text { Vários quar- } \\
\text { teirões }\end{array}$ \\
\hline & $3 \mathrm{i}$ & & & & Andar & & $\begin{array}{l}\text { d } 4500 \text { Andar } \\
\text { distâncias } \\
\text { curtas }\end{array}$ & & Um quarteirão \\
\hline & $3 j$ & & & & $\begin{array}{l}\text { Cuidado } \\
\text { pessoal }\end{array}$ & $\begin{array}{l}\text { Tomar } \\
\text { banho/ } \\
\text { Vestir-se }\end{array}$ & $\begin{array}{l}\text { d5 Cuidado } \\
\text { pessoal }\end{array}$ & $\begin{array}{l}\text { d510 La- } \\
\text { var-se/ d540 } \\
\text { Vestir-se } \\
\end{array}$ & \\
\hline \multirow{2}{*}{$\begin{array}{l}\text { Durante as } \\
\text { últimas } 4 \\
\text { semanas, você } \\
\text { teve algum } \\
\text { dos seguintes } \\
\text { problemas } \\
\text { com seu tra- } \\
\text { balho ou com } \\
\text { alguma ativi- } \\
\text { dade regular, } \\
\text { como conse- } \\
\text { quência de sua } \\
\text { saúde física? }\end{array}$} & $4 \mathrm{a}$ & \multirow[t]{2}{*}{ Avaliação } & \multirow[t]{2}{*}{ Sim ou Não } & \multirow[t]{2}{*}{$\begin{array}{l}\text { Confir- } \\
\text { mação }\end{array}$} & $\begin{array}{l}\text { Saúde } \\
\text { física }\end{array}$ & $\begin{array}{l}\text { Trabalho/ } \\
\text { Ativida- } \\
\text { des regu- } \\
\text { lares }\end{array}$ & nd-sf & $\begin{array}{l}\text { d850 Tra- } \\
\text { balho remu- } \\
\text { nerado/ d230 } \\
\text { Realizar a } \\
\text { rotina diária }\end{array}$ & $\begin{array}{l}\text { Diminui a } \\
\text { quantidade de } \\
\text { tempo }\end{array}$ \\
\hline & $4 \mathrm{~b}$ & & & & $\begin{array}{l}\text { Saúde } \\
\text { física }\end{array}$ & $\begin{array}{l}\text { Trabalho/ } \\
\text { Ativida- } \\
\text { des regu- } \\
\text { lares }\end{array}$ & nd-sf & $\begin{array}{l}\text { d850 Tra- } \\
\text { balho remu- } \\
\text { nerado/ d230 } \\
\text { Realizar a } \\
\text { rotina diária }\end{array}$ & $\begin{array}{l}\text { Realizou me- } \\
\text { nos tarefas }\end{array}$ \\
\hline
\end{tabular}

continua

Outro ponto importante a ser destacado sobre o refinamento diz respeito à regra 4 , que permite identificar as perspectivas relacionadas à proposta de coleta de informações. O guia metodológico propõe alguns exemplos usados com mais frequência, como perspectiva descritiva, que se refere à capacidade ou dificuldade em realizar uma atividade; avaliação, que se refere a questões sobre até que ponto as expectativas pessoais foram alcançadas; e necessidade ou dependência, que se refere ao tipo e nível de necessidade que o indivíduo requer. Além disso, propõe que os itens identificados como perspectiva descritiva sejam diferenciados em capacidade e desempenho ${ }^{10}$. 
Quadro 2. Extração dos conteúdos do SF-36.

\begin{tabular}{|c|c|c|c|c|c|c|c|c|c|}
\hline Cabeçalho & Item & $\begin{array}{c}\text { Pers- } \\
\text { pectiva } \\
\text { adotada } \\
\text { na infor- } \\
\text { mação }\end{array}$ & $\begin{array}{l}\text { Opções de } \\
\text { resposta }\end{array}$ & \begin{tabular}{|c} 
Classifi- \\
cação de \\
opções \\
de res- \\
posta \\
\end{tabular} & $\begin{array}{c}\text { Conceito } \\
\text { princi- } \\
\text { pal }\end{array}$ & $\begin{array}{l}\text { Conceito } \\
\text { adicional }\end{array}$ & $\begin{array}{l}\text { Categoria } \\
\text { CIF do con- } \\
\text { ceito prin- } \\
\text { cipal }\end{array}$ & $\begin{array}{l}\text { Categorias } \\
\text { CIF dos } \\
\text { demais con- } \\
\text { ceitos }\end{array}$ & $\begin{array}{c}\text { Informação } \\
\text { Adicional }\end{array}$ \\
\hline & $4 c$ & \multirow[t]{2}{*}{$\begin{array}{l}\text { Avaliação } \\
\text { Des- } \\
\text { critiva: } \\
\text { Desem- } \\
\text { penho }\end{array}$} & & & $\begin{array}{l}\text { Saúde } \\
\text { física }\end{array}$ & $\begin{array}{l}\text { Trabalho/ } \\
\text { Ativida- } \\
\text { des regu- } \\
\text { lares }\end{array}$ & nd-sf & $\begin{array}{l}\text { d850 Tra- } \\
\text { balho remu- } \\
\text { nerado/ d230 } \\
\text { Realizar a } \\
\text { rotina diária } \\
\end{array}$ & $\begin{array}{l}\text { Esteve lim- } \\
\text { itado }\end{array}$ \\
\hline & $4 d$ & & & & $\begin{array}{l}\text { Saúde } \\
\text { física }\end{array}$ & $\begin{array}{l}\text { Trabalho/ } \\
\text { Ativida- } \\
\text { des regu- } \\
\text { lares }\end{array}$ & nd-sf & $\begin{array}{l}\text { d850 Tra- } \\
\text { balho remu- } \\
\text { nerado/ d230 } \\
\text { Realizar a } \\
\text { rotina diária }\end{array}$ & Estorço extra \\
\hline \multirow{3}{*}{$\begin{array}{l}\text { Durante as } \\
\text { últimas } 4 \text { sem- } \\
\text { anas, você teve } \\
\text { algum dos se- } \\
\text { guintes prob- } \\
\text { lemas com seu } \\
\text { trabalho ou } \\
\text { outra atividade } \\
\text { regular diária, } \\
\text { como con- } \\
\text { sequência de } \\
\text { algum proble- } \\
\text { ma emocional } \\
\text { (como se sen- } \\
\text { tir deprimido } \\
\text { ou ansioso)? }\end{array}$} & $5 a$ & \multirow[t]{2}{*}{ Avaliação } & \multirow[t]{3}{*}{ Sim ou Não } & \multirow[t]{3}{*}{$\begin{array}{l}\text { Confir- } \\
\text { mação }\end{array}$} & $\begin{array}{l}\text { Proble- } \\
\text { ma emo- } \\
\text { cional }\end{array}$ & $\begin{array}{l}\text { Trabalho/ } \\
\text { Ativida- } \\
\text { des regu- } \\
\text { lares }\end{array}$ & nd-sm & $\begin{array}{l}\text { d850 Tra- } \\
\text { balho remu- } \\
\text { nerado/ d230 } \\
\text { Realizar a } \\
\text { rotina diária } \\
\end{array}$ & $\begin{array}{l}\text { Deprimido/ } \\
\text { ansioso }\end{array}$ \\
\hline & $5 b$ & & & & \begin{tabular}{|l} 
Proble- \\
ma emo- \\
cional
\end{tabular} & $\begin{array}{l}\text { Trabalho/ } \\
\text { Ativida- } \\
\text { des regu- } \\
\text { lares }\end{array}$ & nd-sm & $\begin{array}{l}\text { d850 Tra- } \\
\text { balho remu- } \\
\text { nerado/ d230 } \\
\text { Realizar a } \\
\text { rotina diária } \\
\end{array}$ & $\begin{array}{l}\text { Realizou me- } \\
\text { nos tarefas }\end{array}$ \\
\hline & $5 c$ & $\begin{array}{l}\text { Des- } \\
\text { critiva: } \\
\text { Desem- } \\
\text { penho }\end{array}$ & & & $\begin{array}{l}\text { Proble- } \\
\text { ma emo- } \\
\text { cional }\end{array}$ & $\begin{array}{l}\text { Trabalho/ } \\
\text { Ativida- } \\
\text { des regu- } \\
\text { lares }\end{array}$ & nd-sm & $\begin{array}{l}\text { d850 Tra- } \\
\text { balho remu- } \\
\text { nerado/ d230 } \\
\text { Realizar a } \\
\text { rotina diária }\end{array}$ & $\begin{array}{l}\text { Com tanto } \\
\text { cuidado como } \\
\text { geralmente }\end{array}$ \\
\hline $\begin{array}{l}\text { Durante as } \\
\text { últimas } 4 \text { se- } \\
\text { manas, de que } \\
\text { maneira sua } \\
\text { saúde física } \\
\text { ou problemas } \\
\text { emocionais } \\
\text { interferiram } \\
\text { nas suas ativ- } \\
\text { idades sociais } \\
\text { normais, } \\
\text { em relação à } \\
\text { família, amigos } \\
\text { ou em grupo? }\end{array}$ & 6 & $\begin{array}{l}\text { Des- } \\
\text { critiva: } \\
\text { Desem- } \\
\text { penho }\end{array}$ & $\begin{array}{l}\text { De forma } \\
\text { nenhuma } \\
(1) ; \\
\text { Ligeiramente } \\
(2) ; \\
\text { Moderada- } \\
\text { mente (3); } \\
\text { Bastante (4); } \\
\text { Extrema- } \\
\text { mente (5). }\end{array}$ & $\begin{array}{l}\text { Intensi- } \\
\text { dade }\end{array}$ & $\begin{array}{l}\text { Ativi- } \\
\text { dades } \\
\text { sociais } \\
\text { normais }\end{array}$ & \begin{tabular}{|l} 
Saúde \\
física / \\
Proble- \\
mas emo- \\
cionais
\end{tabular} & $\begin{array}{l}\text { d750 Rela- } \\
\text { ções sociais } \\
\text { informais }\end{array}$ & $\begin{array}{l}\text { nd-sf / nd } \\
\text {-sm }\end{array}$ & \begin{tabular}{|l} 
Família, \\
amigos, em \\
grupo
\end{tabular} \\
\hline
\end{tabular}

No presente estudo, a perspectiva da EVA foi identificada como avaliação, para o RMDQ como descritivo de desempenho em 22 itens e dependência nos outros dois, e para o SF-36 como descritivo de desempenho em 25 itens e avaliação em 11 deles.
Em relação à regra cinco, a categorização das opções de resposta do instrumento pode ser descrita como intensidade, frequência, duração, confirmação ou concordância e atributos quali$\operatorname{tativos}^{10}$. Uma área que pode se beneficiar dessa categorização é a prática clínica, que pode dire- 
Quadro 2. Extração dos conteúdos do SF-36.

\begin{tabular}{|c|c|c|c|c|c|c|c|c|c|}
\hline Cabeçalho & Item & $\begin{array}{c}\text { Pers- } \\
\text { pectiva } \\
\text { adotada } \\
\text { na infor- } \\
\text { mação }\end{array}$ & $\begin{array}{l}\text { Opções de } \\
\text { resposta }\end{array}$ & \begin{tabular}{|c|} 
Classifi- \\
cação de \\
opções \\
de res- \\
posta \\
\end{tabular} & $\begin{array}{c}\text { Conceito } \\
\text { princi- } \\
\text { pal }\end{array}$ & $\begin{array}{l}\text { Conceito } \\
\text { adicional }\end{array}$ & $\begin{array}{l}\text { Categoria } \\
\text { CIF do con- } \\
\text { ceito prin- } \\
\text { cipal }\end{array}$ & $\begin{array}{l}\text { Categorias } \\
\text { CIF dos } \\
\text { demais con- } \\
\text { ceitos }\end{array}$ & $\begin{array}{c}\text { Informação } \\
\text { Adicional }\end{array}$ \\
\hline $\begin{array}{l}\text { Quanta dor } \\
\text { no corpo você } \\
\text { teve durante } \\
\text { as últimas } 4 \\
\text { semanas? }\end{array}$ & 7 & $\begin{array}{l}\text { Des- } \\
\text { critiva: } \\
\text { Desem- } \\
\text { penho }\end{array}$ & $\begin{array}{l}\text { Nenhuma } \\
(1) ; \\
\text { Muito leve } \\
(2) ; \\
\text { Leve (3); } \\
\text { Moderada } \\
(4) ; \\
\text { Grave (5); } \\
\text { Muito grave } \\
\text { (6). }\end{array}$ & & $\begin{array}{l}\text { Dor no } \\
\text { corpo }\end{array}$ & & b280 Dor & & \\
\hline $\begin{array}{l}\text { Durante as } \\
\text { últimas } 4 \text { sem- } \\
\text { anas, quanto a } \\
\text { dor interferiu } \\
\text { com seu tra- } \\
\text { balho normal } \\
\text { (incluindo o } \\
\text { trabalho den- } \\
\text { tro de casa)? }\end{array}$ & 8 & Avaliação & $\begin{array}{l}\text { De maneira } \\
\text { alguma (1); } \\
\text { Um pouco } \\
(2) ; \\
\text { Moderada- } \\
\text { mente (3); } \\
\text { Bastante (4); } \\
\text { Extrema- } \\
\text { mente (5). }\end{array}$ & & \begin{tabular}{|l|} 
Interfe- \\
rência da \\
dor
\end{tabular} & & b280 Dor & & \\
\hline $\begin{array}{l}\text { Estas questões } \\
\text { são sobre como } \\
\text { você se sente } \\
\text { e como tudo } \\
\text { tem acon- } \\
\text { tecido com } \\
\text { você durante } \\
\text { as últimas } 4 \\
\text { semanas. Para } \\
\text { cada questão, } \\
\text { por favor dê } \\
\text { uma resposta } \\
\text { que mais se } \\
\text { aproxime de } \\
\text { maneira como } \\
\text { você se sente, } \\
\text { em relação às } \\
\text { últimas } 4 \text { sem- } \\
\text { anas. }\end{array}$ & $9 a$ & $\begin{array}{l}\text { Des- } \\
\text { critiva: } \\
\text { Desem- } \\
\text { penho }\end{array}$ & $\begin{array}{l}\text { All of the } \\
\text { time (1); } \\
\text { Most of the } \\
\text { time (2); } \\
\text { A good bit of } \\
\text { the time (3); } \\
\text { Some of the } \\
\text { time (4); } \\
\text { A little of the } \\
\text { time (5); } \\
\text { None of the } \\
\text { time (6). }\end{array}$ & \begin{tabular}{|l} 
Todo \\
Tempo \\
$(1) ;$ \\
A maior \\
parte do \\
tempo \\
$(2) ;$ \\
Uma boa \\
parte do \\
tempo \\
$(3) ;$ \\
Alguma \\
parte do \\
tempo \\
$(4) ;$ \\
Uma \\
pequena \\
parte do \\
tempo \\
$(5) ;$ \\
Nunca \\
$(6)$.
\end{tabular} & $\begin{array}{l}\text { Sinta-se } \\
\text { cheio de } \\
\text { vigor, } \\
\text { vontade, } \\
\text { força }\end{array}$ & & $\begin{array}{l}\text { b1300 Nível } \\
\text { de energia }\end{array}$ & & \\
\hline
\end{tabular}

continua

cionar a escolha de um instrumento, agora com respeito não apenas ao seu conteúdo, mas também às características das respostas que melhor atendem às suas finalidades.

Nas regras de ligação anteriores ${ }^{6,11}$, foi recomendado que a tabela de resultados incluísse conceitos significativos, categorias da CIF liga- das aos conceitos e informações adicionais. No entanto, o refinamento das regras de vinculação da CIF recomendou que a tabela com os resultados contivesse o nome do instrumento ou outro identificador, a expressão literal da informação de saúde, a perspectiva adotada nas informações, as opções de resposta, a classificação das opções 
Quadro 2. Extração dos conteúdos do SF-36.

\begin{tabular}{|c|c|c|c|c|c|c|c|c|c|}
\hline Cabeçalho & Item & $\begin{array}{c}\text { Pers- } \\
\text { pectiva } \\
\text { adotada } \\
\text { na infor- } \\
\text { mação }\end{array}$ & $\begin{array}{l}\text { Opções de } \\
\text { resposta }\end{array}$ & $\begin{array}{c}\text { Classifi- } \\
\text { cação de } \\
\text { opções } \\
\text { de res- } \\
\text { posta } \\
\end{array}$ & $\begin{array}{c}\text { Conceito } \\
\text { princi- } \\
\text { pal }\end{array}$ & $\begin{array}{l}\text { Conceito } \\
\text { adicional }\end{array}$ & $\begin{array}{l}\text { Categoria } \\
\text { CIF do con- } \\
\text { ceito prin- } \\
\text { cipal }\end{array}$ & $\begin{array}{l}\text { Categorias } \\
\text { CIF dos } \\
\text { demais con- } \\
\text { ceitos }\end{array}$ & $\begin{array}{c}\text { Informação } \\
\text { Adicional }\end{array}$ \\
\hline & $9 \mathrm{~b}$ & & & & Nervoso & & \begin{tabular}{|l} 
b152 \\
Funções \\
emocionais \\
(nervoso)
\end{tabular} & & Muito \\
\hline & $9 c$ & & & & $\begin{array}{l}\text { Depri- } \\
\text { mido }\end{array}$ & & \begin{tabular}{|l|} 
b152 \\
Funções \\
emocionais \\
(deprimido)
\end{tabular} & & \\
\hline & $9 d$ & & & & $\begin{array}{l}\text { Calmo } \\
\text { ou tran- } \\
\text { quilo }\end{array}$ & & $\begin{array}{l}\text { b1263 Es- } \\
\text { tabilidade } \\
\text { psíquica }\end{array}$ & & \\
\hline & $9 e$ & & & & $\begin{array}{l}\text { Com } \\
\text { energia }\end{array}$ & & $\begin{array}{l}\text { b1300 Nível } \\
\text { de energia } \\
\text { (com ener- } \\
\text { gia) }\end{array}$ & & \\
\hline & $9 f$ & & & & \begin{tabular}{|l|} 
Desani- \\
mado ou \\
abatido
\end{tabular} & & $\begin{array}{l}\text { b1265 Oti- } \\
\text { mismo }\end{array}$ & & \\
\hline & $9 g$ & & & & Esgotado & & $\begin{array}{l}\text { b1300 Nível } \\
\text { de energia } \\
\text { (esgotado) }\end{array}$ & & \\
\hline & $9 \mathrm{~h}$ & & & & Feliz & & $\begin{array}{l}\text { b152 Fun- } \\
\text { ções emocio- } \\
\text { nais }\end{array}$ & & \\
\hline & $9 \mathrm{i}$ & & & & \begin{tabular}{|l|} 
Cansado \\
\end{tabular} & & $\begin{array}{l}\text { b1300 Nível } \\
\text { de energia } \\
\text { (cansado) }\end{array}$ & & \\
\hline $\begin{array}{l}\text { Durante as } \\
\text { últimas } 4 \text { se- } \\
\text { manas, quanto } \\
\text { de seu tempo } \\
\text { a sua saúde } \\
\text { física ou prob- } \\
\text { lemas emocio- } \\
\text { nais interfer- } \\
\text { iram com as } \\
\text { suas atividades } \\
\text { sociais (como } \\
\text { visitar amigos, } \\
\text { parentes, etc)? }\end{array}$ & 10 & & $\begin{array}{l}\text { Todo Tempo } \\
(1) ; \\
\text { A maior par- } \\
\text { te do tempo } \\
(2) ; \\
\text { Alguma par- } \\
\text { te do tempo } \\
(3) ; \\
\text { Uma } \\
\text { pequena } \\
\text { parte do } \\
\text { tempo (4); } \\
\text { Nenhuma } \\
\text { parte do } \\
\text { tempo (5). }\end{array}$ & & $\begin{array}{l}\text { Ativi- } \\
\text { dades } \\
\text { sociais }\end{array}$ & $\begin{array}{l}\text { Saúde } \\
\text { física / } \\
\text { Proble- } \\
\text { mas emo- } \\
\text { cionais }\end{array}$ & $\begin{array}{l}\text { d9205 Socia- } \\
\text { lização }\end{array}$ & $\begin{array}{l}\text { nd-sf / nd } \\
\text {-sm }\end{array}$ & $\begin{array}{l}\text { (Visitar ami- } \\
\text { gos, parentes, } \\
\text { etc) }\end{array}$ \\
\hline
\end{tabular}

de resposta, o conceito principal, os conceitos adicionais contidos nas informações, e categoria CIF do conceito principal e a categoria CIF de outros conceitos e anotações. $\mathrm{O}$ item de ano- tação, proposto no refinamento, corresponde às informações adicionais das regras de $2005^{10}$.

Quanto à estruturação dos instrumentos RMDQ e SF-36 percebe-se que enquanto no SF- 
Quadro 2. Extração dos conteúdos do SF-36.

\begin{tabular}{|c|c|c|c|c|c|c|c|c|c|}
\hline Cabeçalho & Item & \begin{tabular}{|c|} 
Pers- \\
pectiva \\
adotada \\
na infor- \\
mação \\
\end{tabular} & $\begin{array}{l}\text { Opções de } \\
\text { resposta }\end{array}$ & \begin{tabular}{|c|} 
Classifi- \\
cação de \\
opções \\
de res- \\
posta \\
\end{tabular} & $\begin{array}{c}\text { Conceito } \\
\text { princi- } \\
\text { pal }\end{array}$ & $\begin{array}{l}\text { Conceito } \\
\text { adicional }\end{array}$ & $\begin{array}{l}\text { Categoria } \\
\text { CIF do con- } \\
\text { ceito prin- } \\
\text { cipal }\end{array}$ & $\begin{array}{l}\text { Categorias } \\
\text { CIF dos } \\
\text { demais con- } \\
\text { ceitos }\end{array}$ & $\begin{array}{c}\text { Informação } \\
\text { Adicional }\end{array}$ \\
\hline \multirow{4}{*}{$\begin{array}{l}\text { O quanto } \\
\text { verdadeiro ou } \\
\text { falso é cada } \\
\text { uma das afir- } \\
\text { mações para } \\
\text { você? }\end{array}$} & $11^{\mathrm{a}}$ & \multirow[t]{4}{*}{ Avaliação } & \multirow{4}{*}{$\begin{array}{l}\text { Definitiva- } \\
\text { mente verda- } \\
\text { deiro (1); } \\
\text { A maioria } \\
\text { das vezes } \\
\text { verdadeiro } \\
(2) \text {; } \\
\text { Não sei (3); } \\
\text { A maioria } \\
\text { das vezes } \\
\text { falso (4); } \\
\text { Definitiva- } \\
\text { mente falso } \\
\text { (5). }\end{array}$} & & Adoecer & & nd-cs & & $\begin{array}{l}\text { Mais facil- } \\
\text { mente que } \\
\text { outras pessoas }\end{array}$ \\
\hline & $11 \mathrm{~b}$ & & & & $\begin{array}{l}\text { Ser sau- } \\
\text { dável }\end{array}$ & & nd-sg & & $\begin{array}{l}\text { Quanto qual- } \\
\text { quer pessoa } \\
\text { que conheço }\end{array}$ \\
\hline & $11 \mathrm{c}$ & & & & Saúde & & nd-sg & & Vai piorar \\
\hline & $11 d$ & & & & Saúde & & nd-sg & & Excelente \\
\hline
\end{tabular}

Tabela 1. Conceitos significativos do SF-36 e do RMDQ ligados aos casos especiais.

\begin{tabular}{|c|c|c|}
\hline Instrumento & Conceito significativo & $\begin{array}{c}\text { Outras } \\
\text { atribuições }\end{array}$ \\
\hline RMDQ & Ficar em casa & $\mathrm{nc}$ \\
\hline SF-36 & Saúde geral & nd-sg \\
\hline SF-36 & Saúde física & nd-sf \\
\hline SF-36 & Atividades vigorosas & nd \\
\hline SF-36 & Atividades moderadas & nd \\
\hline SF-36 & Problemas emocionais & nd-sm \\
\hline SF-36 & Sentir & nd-sm \\
\hline SF-36 & $\begin{array}{l}\text { Minha saúde é } \\
\text { excelente }\end{array}$ & nd-sg \\
\hline SF-36 & $\begin{array}{l}\text { Parece estar um pouco } \\
\text { melhor }\end{array}$ & $\mathrm{nc}$ \\
\hline SF-36 & $\begin{array}{l}\text { Eu sou tão saudável } \\
\text { quanto qualquer pessoa } \\
\text { que conheço }\end{array}$ & $\mathrm{nc}$ \\
\hline SF-36 & $\begin{array}{l}\text { Eu acho que minha } \\
\text { saúde vai piorar }\end{array}$ & $\mathrm{nc}$ \\
\hline $\begin{array}{l}\text { nc: não coberto } \\
\text { não definível - s } \\
\text { nd-sm: não defi } \\
\text { Fonte: as autora }\end{array}$ & $\begin{array}{l}\text { úde } \text { cIF; nd: não definível nd-sf: não definível } \\
\text { ível - saúde mental. }\end{array}$ & $\begin{array}{l}\text { CIF; nd-sg: } \\
\text { - saúde física; }\end{array}$ \\
\hline
\end{tabular}

36, a intensidade e a frequência das dificuldades são diluídas nas respostas, no RMDQ, isso está contido nos itens. Portanto, as informações adicionais contidas nas anotações de conteúdo dos itens do RMDQ se referem à intensidade e frequ- ência das dificuldades, enquanto as informações adicionais do SF-36 se referem a exemplos e especificações dos itens.

A proposta de ligar esses três instrumentos utilizados na pesquisa e na prática clínica para avaliação da dor crônica foi reforçada com a aplicação dos refinamentos das regras de vinculação, dando maior clareza aos resultados expressos nos Quadros 1 e 2 com as colunas dos conceitos principais, conceitos adicionais e categorias a eles associados.

Observando os estudos anteriores e o presente estudo, houve um aumento gradual tanto do número de conceitos identificados quanto das categorias ligadas ao longo das publicações dos instrumentos SF-36 6,11,15,17-19 (Tabela 3) e $\mathrm{RMDQ}^{16}$. Acredita-se que a identificação de mais conceitos significativos no presente estudo tenha ocorrido devido à consideração das informações nos cabeçalhos e respostas das questões. O número de categorias ligadas neste estudo em comparação com o número encontrado em estudos anteriores foi maior, mas não tão expressivo. Essa observação corrobora o objetivo da proposta de aprimoramento para melhorar a captura de dados e as nuances das informações, melhorando a exposição dos dados.

Ao analisar estudos prévios ao refinamento, que vincularam o instrumento SF-36,11,15,17-19,30 (Tabela 3) e o RMDQ ${ }^{16}$, observou-se que apenas 
Tabela 2. Número de componentes e categorias selecionadas.

\begin{tabular}{lcccc}
\hline & \multicolumn{2}{c}{ RMDQ (24 itens) } & \multicolumn{2}{c}{ SF-36 (36 itens) } \\
\cline { 2 - 5 } & $\begin{array}{c}\text { Conceito } \\
\text { principal }\end{array}$ & $\begin{array}{c}\text { Conceito } \\
\text { Adicional }\end{array}$ & $\begin{array}{c}\text { Conceito } \\
\text { principal }\end{array}$ & $\begin{array}{c}\text { Conceito } \\
\text { Adicional }\end{array}$ \\
\hline Conceitos ligados a componentes da CIF & & & & \\
Funções do Corpo & 24 & 3 & 11 & 0 \\
Estruturas do Corpo & 0 & 0 & 0 & 0 \\
Atividades e Participação & 0 & 19 & 12 & 0 \\
Fatores Ambientais & 0 & 4 & 0 & 0 \\
Conceitos nd-sg & 0 & 0 & 5 & 2 \\
Conceitos nd-sf & 0 & 0 & 4 & 2 \\
Conceitos nd-sm & 0 & 0 & 3 & 0 \\
Conceitos nd-cs & 0 & 0 & 1 & 30 \\
Conceitos nc & 0 & 1 & 0 & 0 \\
Total de conceitos & 24 & 27 & 36 & 0 \\
Número de categorias da CIF por & & & & 0 \\
componente sem duplicação & & & & 0 \\
Funções do Corpo & 1 & 3 & 5 & 13 \\
Estruturas do Corpo & 0 & 0 & 0 & 0 \\
Atividades e Participação & 0 & 15 & 9 & 0 \\
Fatores Ambientais & 0 & 2 & 14 & \\
Total & 1 & 20 & & \\
\hline Fis & & & & \\
\hline
\end{tabular}

Fonte: as autoras.

Cieza et al. ${ }^{11}$ expuseram os conceitos significativos identificados em todos os instrumentos e a ligação de todos eles à CIF. Cieza et al. ${ }^{6}$, Geyh et al. ${ }^{19}$, Schepers et al. ${ }^{18}$, Prodinger et al. ${ }^{15}$ e Sigl et al. ${ }^{16}$ mostraram as categorias selecionadas, mas não mostraram a qual item de instrumento estavam ligados. Scheuringer et al. ${ }^{30}$ ligaram o conteúdo de 120 instrumentos, entre eles o SF-36, mas os resultados expressaram o total de categorias relacionadas a todos os instrumentos. Fréz et al. ${ }^{17}$ ligaram apenas os domínios do SF-36 às categorias do CIF.

Acredita-se que o detalhamento dos resultados esteja relacionado ao objetivo do estudo e ao número de instrumentos relacionados, quanto maior o número de instrumentos ligados, menor a possibilidade de detalhamento.

Rat et al. ${ }^{31}$ e Milman et al. ${ }^{32}$ usaram as ligações já estabelecidas do SF-36 para comparar o conteúdo com outros instrumentos. Faria et al. ${ }^{33}$ realizaram uma revisão sistemática para identificar categorias do componente Atividades e Participação em alguns instrumentos de qualidade de vida já ligados à CIF. Isso mostra a importância de ligar esses questionários usando o refinamento de regras, para que também possa servir de base de comparação para o estabelecimento de outros instrumentos.
Como o objetivo deste trabalho foi relatar a experiência de aplicar o refinamento das regras de ligação, o estudo apresenta os resultados detalhados sugeridos por Cieza et al. ${ }^{10}$, permitindo que os usuários acessem informações sobre o conteúdo desses instrumentos de medição, o que pode auxiliar profissionais e pesquisadores na escolha dos instrumentos que melhor atendam seus interesses. Esta situação pode ser explicada com a EVA. No presente estudo, a categoria b280 foi escolhida, mas, dependendo do contexto da pesquisa, pode ser escolhida uma categoria mais específica relacionada à dor, como lombalgia, a categoria b28013.

Algumas possíveis limitações do presente estudo devem ser consideradas, mesmo que informações importantes tenham sido obtidas para a operacionalização do refinamento das regras de ligação e sua divulgação. Observaram-se diferenças nos achados gerais de estudos que utilizaram os mesmos instrumentos, usando as mesmas regras. Essa situação mostra que a experiência e o contexto clínico devem ser considerados ao selecionar a versão que melhor atenda às suas necessidades. Também é necessário que outros profissionais usem as novas regras de ligação em diferentes contextos clínicos para identificar sua contribuição para a produção de registros de 
funcionalidade e monitoramento de estados de saúde.

Uma limitação para a discussão dos resultados foi a falta de identificação clara nos estudos cujos conceitos foram identificados e seus respectivos itens nos instrumentos investigados. Nesses casos, os interessados em usar os estudos como referência devem seguir o outro caminho para identificar o que está vinculado.

Dentre os estudos incluídos neste estudo, apenas Cieza et al. ${ }^{11}$ apresentaram clareza na apresentação dos achados, portanto, acredita-se que a recente inserção de uma tabela de exposição dos resultados ajude trabalhos futuros, bem como o registro dos construtos capacidade e desempenho considerados recentemente. Dessa forma, o profissional/pesquisador que utilizará os instrumentos poderá conhecer os itens associados às categorias da CIF, sua especificação ou conteúdo.

Sabe-se que esforços internacionais foram aplicados para associar instrumentos validados às categorias da CIF. Cieza et al. ${ }^{10}$ sugerem que o uso continuado das regras atualizadas para vincular instrumentos à CIF pode ajudar na criação de um banco de dados contendo conjuntos de itens ligados a cada categoria da CIF, visando tanto o desenvolvimento de novos instrumentos quanto a operacionalização de certas categorias da CIF e instrumentos de acordo com a perspectiva que melhor se adequa ao objetivo clínico. Este exercício também fornecerá informações para especificar melhor as categorias relevantes para uma futura revisão de classificação.

$\mathrm{O}$ uso da CIF instrumentaliza profissionais na prática clínica, bem como profissionais de programas e políticas públicas, responsáveis por avaliações e classificações de funcionalidade e incapacidade ${ }^{8}$. Seu uso também poderia ajudar a selecionar e construir instrumentos de conteúdos mais precisos e em fonte quantitativa de informação ${ }^{10}$.

Assim, é importante que mais estudos como esse sejam realizados para expandir as bases de dados e avaliar a influência das regras de ligação refinadas no processo de ligar instrumentos com diferentes perspectivas e diferentes categorias de respostas.

\section{Conclusão}

O refinamento das regras de ligação proporcionou maior clareza no processo de identificar e ligar o conteúdo dos instrumentos com a CIF e 
expor os resultados. Assim, aumentou o número de conceitos identificados e ligados, bem como o número de categorias nos instrumentos.

O refinamento das regras permite a padronização de conceitos de instrumentos de medida e da CIF em diferentes níveis de precisão, com a especificação/identificação da perspectiva e da categorização das respostas. Os resultados expressos em uma tabela com as informações principais sobre o processo de ligação irão ajudar a aumentar a transparência e a confiabilidade bem como a comparação entre estudos.

O conteúdo de ligação de diversos instrumentos como EVA, RMDQ, e SF-36 baseados no refinamento das regras contribuirá para o alinhamento dos instrumentos existentes com a CIF e com a seleção de instrumentos que abranjam parâmetros relevantes de interesse de pesquisadores e clínicos.

\section{Colaboradores}

Todos os autores contribuíram fundamentalmente para este estudo. BC Santos trabalhou na pesquisa, design, metodologia e processo de ligação da CIF. KO Scharan, KP Corrêa e RS Bernardelli trabalharam na pesquisa, projeto, metodologia, processo de ligação da CIF, análise de dados e redação do presente estudo. MIB Silveira trabalhou na pesquisa, metodologia e processo de ligação da CIF. ADL Moser trabalhou na concepção e design, orientou o processo de ligação e redação do artigo e fez a revisão final.

\section{Referências}

1. Nascimento PRC, Costa LOP. Prevalência da dor lombar no Brasil: uma revisão sistemática. Cad Saúde Pública 2015; 31(6):1141-1156.

2. Goldberg DS, McGee SJ. Pain as a global public health priority. BMC Public Health 2011; 11(1):770.

3. Garcia JBS, Hernandez-Castro JJ, Nunez RG, Pazos MAR, Aguirre JO, Jreige A, Delgado W, Serpentegui M, Berenguel M, Cantemir C. Prevalence of low back pain in Latin America: a systematic literature review. Pain Physician 2014; 17(5):379-391.

4. Arcanjo GN, Valdés MTM, Silva RM. Percepção sobre qualidade de vida de mulheres participantes de oficinas educativas para dor na coluna. Cien Saude Colet 2008; 13(Supl. 2):2145-2154.

5. Organização Mundial da Saúde (OMS). Centro Colaborador da Organização Mundial da Saúde para a Família de Classificações Internacionais em Português. In: Buchalla CM, organizador. CIF: Classificação Internacional de Funcionalidade, Incapacidade e Saúde. São Paulo: Editora da Universidade de São Paulo; 2015.

6. Cieza A, Geyh S, Chatterji S, Kostanjsek N, Ustun B, Stucki G. ICF linking rules: an update based on lessons learned. J Rehabil Med 2005; 37(4):212-218.

7. Cieza A, Stucki G. Content comparison of health-related quality of life (HRQOL) instruments based on the international classification of functioning, disability and health (ICF). Quality Life Res 2005; 14(5):1225-1237. 
8. Santos W. Deficiência como restrição de participação social: desafios para avaliação a partir da Lei Brasileira de Inclusão. Cien Saude Colet 2016; 21(10):3007-3015.

9. Neves-Silva P, Álvarez-Martín E. Estudio descriptivo de las características sociodemográficas de la discapacidad en América Latina. Cien Saude Colet 2014; 19(2):4889-4898.

10. Cieza A, Fayed N, Bickenbach J, Prodinger B. Refinements of the ICF Linking Rules to strengthen their potential for establishing comparability of health information. Disabil Rehabil 2019; 41(5):574-583.

11. Cieza A, Brockow T, Ewert T, Amman E, Kollerits B, Chatterji S, Ustün TB, Stucki G. Linking health-status measurements to the international classification of functioning, disability and health. J Rehabil Med 2002; 34(5):205-210.

12. Lukacz ES, Lawrence JM, Burchette RJ, Luber KM, Nager CW, Buckwalter JG. The use of Visual Analog Scale in urogynecologic research: a psychometric evaluation. Am J Obstet Gynecol 2004; 191(1):165-170.

13. Roland M, Morris R. A Study of the Natural History of Low-Back Pain: Part II: Development of Guidelines for Trials of Treatment in Primary Care. Spine 1983; 8(2):145-150.

14. Ciconelli RM, Ferraz MB, Santos W, Meinão I, Quaresma MR. Tradução para a língua portuguesa e validação do questionário genérico de avaliação de qualidade de vida SF-36 (Brasil SF-36). Rev Bras Reumatol 1999; 39(3):143-150.

15. Prodinger B, Cieza A, Williams DA, Mease P, Boonen A, Kerschan-Schindl K, Fialka-Moser V, Smolen J, Stucki G, Machold K, Stamm T. Measuring health in patients with fibromyalgia: content comparison of questionnaires based on the International Classification of Functioning, Disability and Health. Arthritis Rheum 2008; 59(5):650-658.

16. Sigl T, Cieza A, Brockow T, Chatterji S, Kostanjsek N, Stucki G. Content comparison of low back pain-specific measures based on the International Classification of Functioning, Disability and Health (ICF). Clin J Pain 2006; 22(2):147-153.

17. Fréz AR, Abdallah AA, Riedi C, Galindo J, Ruaro JA, Ribeiro SC. Proposed use of the international classification of functioning, disability and health to evaluate quality of life after an amputation. Fisioterapia Movimento 2014; 27(1):49-56.

18. Schepers V, Ketelaar M, Van de Port I, Visser-Meily J, Lindeman E. Comparing contents of functional outcome measures in stroke rehabilitation using the International Classification of Functioning, Disability and Health. Disabil Rehabil 2007; 29(3):221-230.

19. Geyh S, Cieza A, Kollerits B, Grimby G, Stucki G. Content comparison of health-related quality of life measures used in stroke based on the international classification of functioning, disability and health (ICF): a systematic review. Qual Life Res 2007;16(5):833-851.

20. Brennan P, Silman A. Statistical methods for assessing observer variability in clinical measures. BMJ 1992; 304(6840): 1491 .

21. Cohen J. A coefficient of agreement for nominal scales. Educ Psychol Measurement 1960; 20(1):37-46.

22. Feinstein AR, Cicchetti DV. High agreement but low kappa: I. The problems of two paradoxes. J Clinical Epidemiol 1990; 43(6):543-549.
23. Krippendorff K. Reliability. Wiley Online Library; 1980.

24. Campbell JL, Quincy C, Osserman J, Pedersen OK. Coding in-depth semistructured interviews: Problems of unitization and intercoder reliability and agreement. Sociol Methods Res 2013; 42(3):294-320.

25. Miot HA. Análise de concordância em estudos clínicos e experimentais. J Vasc Bras 2016; 15(2):89-92.

26. Rosner B. Fundamentals of biostatistics. USA: Duxbury Press; 1994

27. Stamm T, Geyh S, Cieza A, Machold K, Kollerits B, Kloppenburg M, Smolen J, Stucki G. Measuring functioning in patients with hand osteoarthritis—content comparison of questionnaires based on the International Classification of Functioning, Disability and Health (ICF). Rheumatology 2006; 45(12):1534-1541.

28. Fayed N, Cieza A, Bickenbach JE. Linking health and health-related information to the ICF: a systematic review of the literature from 2001 to 2008. Disabil Rehabil 2011; 33(21-22):1941-1951.

29. Madden RH, Bundy A. The ICF has made a difference to functioning and disability measurement and statistics. Disabil Rehabil 2019; 41(12):1450-1462.

30. Scheuringer M, Grill E, Boldt C, Mittrach R, Müllner P, Stucki G. Systematic review of measures and their concepts used in published studies focusing on rehabilitation in the acute hospital and in early post-acute rehabilitation facilities. Disabil Rehabil 2005; $27(7-$ 8):419-429.

31. Rat A-C, Guillemin F, Pouchot J. Mapping the osteoarthritis knee and hip quality of life (OAKHQOL) instrument to the international classification of functioning, disability and health and comparison to five health status instruments used in osteoarthritis. Rheumatology 2008; 47(11):1719-1725.

32. Milman N, Boonen A, Merkel PA, Tugwell P. Mapping of the Outcome Measures in Rheumatology Core Set for Antineutrophil Cytoplasmic Antibody Associated Vasculitis to the International Classification of Function, Disability and Health. Arthritis Care Res (Hoboken) 2015; 67(2):255-263.

33. Faria CDCM, Silva SM, Corrêa JCF, Laurentino GEC, Teixeira-Salmela LF. Identificação das categorias de participação da CIF em instrumentos de qualidade de vida utilizados em indivíduos acometidos pelo acidente vascular encefálico. Rev Panam Salud Publica 2012; 31(4):339.

Artigo apresentado em 19/09/2017

Aprovado em 07/06/2019

Versão final aprovada em 09/06/2019

Editores-chefes: Romeu Gomes, Antônio Augusto Moura da Silva 